\section{NSCLC: Empfehlung für Gefitinib}

Gefitinib führt als First-Line-Therapie des nichtkleinzelligen Lungenkarzinoms (NSCLC) mit Gefitinib-sensitiven Mutationen zu einem längeren progressionsfreien Überleben als die klassische Kombination aus Carboplatin plus Paclitaxel. Nun liegen auch erste Daten für das Gesamtüberleben vor.

0 b Patienten auf den Tyrosinkinaseinhibitor Gefitinib ansprechen, hängt von speziellen Mutationen in der Kinase-Domäne des epidermalen Wachstumsfaktorrezeptors (EGFR) ab. Mehrere Phase-II-Studien haben gezeigt, dass sich die NSCLC-Therapie auf der Grundlage der EGFR-Mutationen individualisieren lässt. Die North-East Japan Study Group verglich daher in einer Phase-IIIStudie zur First-Line-Therapie des fortgeschrittenen NSCLC mit EGFR-Mutationen Gefitinib mit dem StandardKombinationsregime aus Carboplatin plus Paclitaxel. Die Studie ergab, dass Gefitinib ein signifikant längeres progressionsfreies Überleben ermöglicht als Carboplatin/Paclitaxel und bestätigte damit weitere Studien mit ähnlichen Ergebnissen. Für das fortgeschrittene NSCLC mit sensitiven EGFR-Mutationen gilt deshalb Gefitinib in der FirstLine-Therapie als Standard. Doch viele
Kollegen betrachten diese Empfehlung als kritisch, solange keine Daten für das Gesamtüberleben existieren.

Die North-East Japan Study Group legte nun weitere Beobachtungsdaten ihrer Studie mit 228 Patienten vor, in der das Gesamtüberleben sekundärer Endpunkt war. Das Follow-up umfasst jetzt median 23 Monate statt zuvor 17.

Demnach betrug die mediane Überlebenszeit 27,7 Monate in der GefitinibGruppe und 26,6 Monate in der Carboplatin/Paclitaxel-Gruppe (Hazard Ratio $0,887 ; p=0,483)$. Die Patienten erhielten allerdings nach der First-Line-Therapie noch Second- und Third-Line-Behandlungen, die bei fast allen Carboplatin/Paclitaxel-Patienten Gefitinib beinhalteten.

Die japanischen Kollegen verglichen deshalb das Gesamtüberleben der Patienten, die während ihrer gesamten $\mathrm{Be}$ handlung Platinderivate erhielten $(\mathrm{n}=$ 186) mit denen von Patienten, die nie ein
Platinderivat erhalten hatten $(n=40)$. Es fand sich kein signifikanter Unterschied. Nur zwei Patienten hatten kein Gefitinib erhalten. Die mediane Überlebenszeit von Patienten, die mit Gefitinib, Platinderivaten, Permetrexed oder Docetaxel behandelt worden waren, betrug etwa drei Jahre $(n=76)$.

Fazit: Unter Gefitinib wird zumindest ein ebenbürtiges Gesamtüberleben wie unter der Kombination aus Carboplatin plus Paclitaxel erreicht. Dass Gefitinib nicht überlegen ist, könnte darauf zurückzuführen sein, dass fast alle Patienten aus der Carboplatin/PaclitaxelGruppe für die Second-Line-Therapie zu Gefitinib wechselten. Da zuvor schon gezeigt wurde, dass die Lebensqualität unter Gefitinib erheblich höher ist als unter der klassischen platinbasierten Therapie, solle beim EGFR-mutierten NSCLC first-line weiterhin Gefitinib in der Erstlinie eingesetzt werden, so die Autoren.

Christina Berndt

Inoue A et al. Updated overall survival results from a randomized phase III trial comparing gefitinib with carboplatin-paclitaxel for chemonaïve non-small cell lung cancer with sensitive EGFR gene mutations (NEJ002). Ann Oncol. 2013;24(1):54-9.

\title{
Weniger Lungenkrebstote durch Screening mittels Niedrigdosis-CT
}

\section{Lungenkrebs zählt nach wie vor zu den häufigsten krebsbedingten Todes- ursachen, weil die Erkrankung in der Mehrzahl der Fälle erst in fort- geschrittenem Stadium erkannt wird. Einer aktuellen epidemiologischen Studie zufolge könnte ein Screening mit Niedrigdosis-CT (LDCT) in den USA bis zu 12.000 Personen das Leben retten.}

D er Studie zugrunde liegt das Untersuchungsjahr 2010, in dem etwa 14,3\% der US-Bevölkerung die Kriterien des National Lung Screening Trial (NLST) für ein jährliches Lungenkrebsscreening erfüllten. Insgesamt waren das nahezu 8,6 Millionen Amerikaner. Diese Daten nutzten die Wissenschaftler um den Epidemiologen Jiemin Ma in ihrer Computersimulation.

Vorausgesetzt wurde bei der Berechnung, dass die für das Screening geeigneten Personen zu 100\% teilnehmen.
Aufgrund dieser Annahmen könnte durch ein flächendeckendes LDCTScreening der Tod von insgesamt 12.500 Personen (8.990 Männer und 3.260 Frauen) verhindert werden. Läge der Anteil nur bei $70 \%$ - also etwa so hoch wie beim Mammografiescreening in den USA in den vergangenen zwei Jahren -, könnten der Simulation zufolge noch immer 8.600 Lungenkrebstodesfälle pro Jahr verhindert werden. Zur Erinnerung: In der NLST-Studie mit mehr als 53.000 Teilnehmern starben $20 \%$ weniger Men- schen (356 vs. 443) an den Folgen von Lungenkrebs, wenn sie an einem Screening mit LDCT statt einem Screening mit der konventionellen Röntgenthoraxdiagnostik teilgenommen hatten.

Fazit: Die Tatsache, dass sich die Lungenkrebssterberate durch ein LDCTScreening um $20 \%$ senken lässt und möglicherweise 12.000 Lungenkrebstodesfälle verhindert werden können, reiche nicht aus für dessen Aufnahme in ein nationales Screeningprogramm, so Larry Kessler von der Universität Washington in Seattle, WA/USA, in seinem Kommentar zur Simulationsstudie. Zuvor sei eine klare Risiko-Nutzen-Analyse erforderlich.

Peter Leiner

Ma J et al. Annual Number of Lung Cancer Deaths Potentially Avertable by Screening in the United States. Cancer. 2013;119(7):1381-5 Editorial: Kessler L. Is $20 \%$ of a loaf enough? ibidem 1294-7 